\title{
A THRESHOLD FOR A CARICATURE OF THE NERVE EQUATION
}

\author{
BY H. P. McKEAN ${ }^{1}$ AND V. MOLL
}

1. Introduction. Hodgkin-Huxley $[\mathbf{1 9 5 2}]^{2}$ described the conduction of the nervous impulse in the optical nerve of a squid. ${ }^{3}$ The physiological fact to be modelled is that stimuli below threshold damp out and so convey no information, but a stimulus above threshold is rapidly converted into a train of pulses of approximately fixed shape and spacing that travel down the line with little distortion. Fitzhugh [1961/69] and Nagumo et al. [1964] proposed a simplified model: ${ }^{4}$

$$
\text { (1) } \frac{\partial u}{\partial t}=\frac{\partial^{2} u}{\partial x^{2}}+u(1-u)(u-a)+e^{-c t} f+b e^{-c t} * u, \quad(t, x) \in[0, \infty] \times \mathbf{R},
$$

in which $0<a<1, b \leq 0$ is a (small) coupling, $c>0$ is a damping, and $f$ is (part of the) initial data. McKean [1970] suggested replacing the cubic in (1) by a broken line of the same general shape. The present announcement deals with this caricature:

(2) $\frac{\partial u}{\partial t}=\frac{\partial^{2} u}{\partial x^{2}}-u+(1$ if $u>a)+e^{-c t} f+b e^{-c t} * u, \quad(t, x) \in[0, \infty] \times \mathbf{R}$.

It is believed, but not proven, that (1) and (2) have similar portraits in the large. This would be helpful since (2) is much more tractable than (1). The mathematical problem posed by (2) (or (1)) is to classify its waveforms and to prove that every solution of a suitable initial size and shape, tracked at the proper speed, converges to one of them. The actual diversity of waveforms for (2) is quite staggering, as Feroe [1981] and others have confirmed: For suitable values of $a, b, c,(2)$ admits trains of $1,2,3, \ldots, \infty$ pulses; moreover, the $\infty$-pulse trains come in many varieties. In view of this complexity it is natural to classify the waveforms according to the number of crossings of the level $a$ and to begin with the simplest cases.

2. One crossing: rising waves. McKean $[\mathbf{1 9 8 3} / \mathbf{8 4}]$ dealt with this case for small $b, c>0$ and $a(1-b / c)<1$; the same result was obtained for $b=0$ by Fife-Mcleod [1977] for (1) and by Terman [1983] for (2). The fact is that, up to translation, (2) has a single waveform $w(x)$ which rises from

\footnotetext{
Received by the editors October 30, 1984.

1980 Mathematics Subject Classification. Primary 35K55, 35Q20; Secondary 92A09.

${ }^{1}$ The work presented in this paper was performed at the Courant Institute of Mathematical Sciences, New York, with the support of the National Science Foundation under Grant NSF-MSC-76-07039.

${ }^{2}$ Hodgkin $[\mathbf{1 9 7 1}]$

${ }^{3}$ Cohen [1976], Hadeler [1976], and Rinzel [1976] review the model and its simplifications.

${ }^{4}$ means convolution.
} 
$w(-\infty)=0$ to $w(+\infty)=(1-b / c)^{-1}>a$; it travels at a definite speed $k=k(a, b, c)$ and may be standardized by fixing $w(0)=a$. Now let $u(t, x)$ be any solution of (2) issuing from data of the same general (rising) shape as $w$. Then $u(t, x+k t)=w(x-m)+o(1)$ for $t \uparrow \infty$, as proposed above. McKean $[\mathbf{1 9 8 3} / \mathbf{8 4}]$ proved a little more: The phase shift $m$ is a smooth function of the initial data with nonvanishing gradient, so that the whole space of data (of the present rising shape) is foliated by smooth leaves of codimension 1 distinguished by the associated value of $m$.

3. Two crossings: phase portrait. The next simplest case (two crossings) is the subject of this announcement. A complete picture is obtained, but only for $b=0$ and $c=\infty$, which makes the last two terms drop out of (2). Then (2) admits a waveform $w(x)$ vanishing at $\pm \infty$ with a single peak above the level $a$ provided $a<1 / 2$. The speed vanishes, so $w$ is a standing wave; moreover, it is symmetrical about its peak (which may be placed at $x=0$, by translation) and falls steadily for $x \geq 0$. The flow regulated by (2) preserves the space of data of the same general (symmetrical falling) shape. A technical difficulty must be confessed: If $a$ is small, if the initial datum satisfies $u(0)=a \geq u(x)(x \neq 0)$, and if $b=-u^{\prime \prime}(0) \geq 0$ is also small, then (2) has a lower solution, which falls at once below the level $a$ and follows $\partial u / \partial t=\partial^{2} / \partial x^{2}-u$ to ultimate collapse $[u \equiv 0]$, and also an upper solution, which is generally distinct. This does not happen if $a+b$ exceeds the number

$$
a_{*}=\max _{c>0} \int_{0}^{1} d t \int_{c t^{-1 / 2}(1-\sqrt{1-t})}^{c t^{-1 / 2}(1+\sqrt{1-t})} \frac{e^{-x^{2} / 4}}{\sqrt{4 \pi}} d x<\frac{1}{2}
$$

so it is safest to take $a_{*}<a<1 / 2$. Then the solution of (2) is unambiguous. Now an infinitesimal analysis of (2) about the standing wave reveals the existence of one unstable mode, a spectral gap down to -1 , and continuous spectrum filling $(-\infty,-1)$, indicating the presence of a codimension-1 saddlepoint centered at the standing wave; indeed, this is exactly the phase portrait of (2), not just in the small, but in the large. There is a region of collapsing data for which the solution ultimately falls below the level $a$ and follows $\partial u / \partial t=\partial^{2} u / \partial x^{2}-u$ to the resting state $u(\infty, x) \equiv 0$; a region of expanding data for which the leading (trailing) edge of the solution acts like a falling (rising) wave moving out to $+\infty(-\infty)$, and the resting state $u(\infty, x) \equiv 1$ is achieved; and these two regions are separated by a smooth submanifold of codimension 1 (the threshold) on which every trajectory tends exponentially fast to the standing wave.

3. Proofs. The advantage of (2) over (1) is that the behaviour of $u(t, x)$ for $t \uparrow \infty$ is regulated by its median:

$$
\begin{aligned}
m(t) & =\text { the positive root of } u(t, x)=a \quad \text { if } u(t, 0)>a \\
& =0 \quad \text { if } u(t, 0) \leq a
\end{aligned}
$$


The point is that

$$
\begin{aligned}
u(t, x)= & e^{-t} \int_{-\infty}^{\infty} \frac{e^{-(x-y)^{2} / 4 t}}{\sqrt{4 \pi t}} u_{0}(y) d y \\
& +\int_{0}^{t} e^{-s} d s \int_{x-m(t-s)}^{x+m(t-s)} \frac{e^{-x^{2} / 4 s}}{\sqrt{4 \pi s}} d y
\end{aligned}
$$

in which the first integral is negligible for $t \uparrow \infty$. If $m(t)>0$, then evaluation of $(3)$ at $x=m(t)$ yields a (complicated) integral equation:

$$
a=O\left(e^{-t}\right)+\int_{0}^{t} e^{-s} d s \int_{m(t)-m(t-s)}^{m(t)+m(t-s)} \frac{e^{-x^{2} / 4 s}}{\sqrt{4 \pi s}} d x
$$

This is the chief tool. There are four main steps.

Step 1 is to prove that the median is bounded from 0 and $\infty$ if the solution is critical in the sense that it neither collapses (to $u \equiv 0$ ) nor expands (to $u \equiv 1){ }^{5}$ This provides a compactness to the translates of $m$, which permits the question of the tendency of critical solutions to the standing wave to be reduced to the question of whether

$$
a=\int_{0}^{\infty} e^{-s} d s \int_{m(t)-m(t-s)}^{m(t)+m(t-s)} \frac{e^{-x^{2} / 4 s}}{\sqrt{4 \pi s}} d s
$$

has any other solutions besides the (constant) median $m(t)=m(\infty)$ of the standing wave. The answer is no, it does not, provided $a$ is close to $1 / 2 .^{6}$ The proof is by repeated estimation of $|m(t)-m(\infty)|$ in terms of $|m \cdot(t)|$ and vice versa, the appraisals being ultimately contradictory unless $m(t) \equiv m(\infty)$.

Step 2 is to return to (4) armed with the information that the actual median satisfies $m(t)=m(\infty)+o(1)$ and elicits the sharp estimate $m(t)-m(\infty)=$ $O\left(e^{-t} \sqrt{t}\right)$. This shows, via $(4)$, that $u(t, x)$ tends exponentially fast to the standing wave.

Step 3 is to elicit the existence of a sharp threshold. Let $u(x)$ be any (symmetrical falling) initial datum of (2). The previous remarks about collapse and expansion imply the existence of numbers $0<c_{*} \leq c^{*}<\infty$ such that the solution of (2) issuing from $c u(x)$ collapses for $0<c<c_{*}$, expands for $c^{*}<c<\infty$, and does neither (i.e., is critical) for $c_{*}<c<c^{*}$ if there is a gap $\left(c_{*}<c^{*}\right)$. The existence of such a gap is unreasonable in view of the infinitesimal analysis cited before and the stabilization of critical solutions to the standing wave; in fact, it is nonexistent, so that each ray $\mathbf{R}^{+} u$ contains

\footnotetext{
${ }^{5}$ Terman $[\mathbf{1 9 8 3}]$ proved that, for small $b \leq 0, u$ expands to $u \equiv(1-b / c)^{-1}$ as soon as its initial data lies above the level $a$ in a sufficiently long interval.

${ }^{6}$ The same may be true without the proviso, but the proof escapes us; indeed, there is still the intriguing possibility that (5) admits nonconstant (periodic?) solutions if $a$ is small.
} 
just one critical point. These form the threshold advertised before. The proper value of $c$ is the critical multiplier of $u$; the locus $c=1$ is the threshold.

Step 4 is to prove that the threshold is a smooth manifold of codimension 1 in the ambient space; the fact that $a$ is close to $1 / 2$ is also important here. A self-evident technical difficulty is that (2) is not close to its variational equation in any conventional sense, owing to the fact that the nonlinearity is not smooth. This is overcome by making a detailed estimate of the difference $v=u_{+}-u_{-}$of two neighboring critical solutions of (2). The result is

$$
v=e^{\omega t} A(x) \int_{0}^{\infty} B v_{0} d x+o(1) \quad(t \uparrow \infty)
$$

in which $\omega>0$ is the eigenvalue for the unstable infinitesimal mode cited before, $A>0$ is the associated ground state, and $B>0$ is a closely allied function expressed as a functional integral based upon the diffusion with infinitesimal operator $\partial^{2} / \partial x^{2}+2\left(A^{\prime} / A\right)(\partial / \partial x)(x \geq 0)$, subject to reflection at $x=0$. Now $u_{+}$and $u_{-}$are critical, so $v=o(1)$ as $t \uparrow \infty$ and $\int_{0}^{\infty} B v_{0}=0$, by (6). The smoothness of the threshold is deduced from that by identifying the gradient of the critical multiplier $c$ on the threshold with the limit of $-B\left[\int_{0}^{\infty} B u_{0}\right]^{-1}$ as the data $u_{+0}$ and $u_{-0}$ are brought into coincidence. This shows that $c$ is smooth on threshold and, since it is a homogeneous function of degree -1 , off threshold, so it is smooth in the large, and the threshold $(c=1)$ inherits that property. This was the aim of Step 4 . The details will be published elsewhere.

\section{BIBLIOGRAPHY}

H. Cohen, Mathematical developments in Hodgkin-Huxley theory and its approximations, Lectures Math. Life Sci., Vol. 8, Amer. Math. Soc., Providence, R.I., 1976, pp. 89-124.

J. Feroe, Travelling waves of infinitely many pulses in nerve equations, Math. Biosci. 55 (1981), 189-203.

P. Fife and J. McLeod, The approach of solutions of non-linear diffusion equations to travelling front solutions, Arch. Rational Mech. Anal. 65 (1977), 335-361.

R. Fitzhugh, Impulses and physiological states in theoretical models of nerve membrane, Biophys. J. 1 (1961), 445-466.

, Mathematical models of excitation and propagation in nerve, Biological Engineering (H. Schwan, ed.), 1969, pp. 1-85. 206.

K. Hadeler, Non-linear diffusion equations in biology, Lect. Notes in Math. 564 (1976), 1631971.

A. L. Hodgkin, The conduction of the nervous impulse, Liverpool Univ. Press, Liverpool,

A. L. Hodgkin and A. F. Huxley, A qualitative description of membrane current and its application to conduction and excitation in nerve, J. Physiol. 117 (1952), 500-544.

H. P. McKean, Nagumo's equation, Adv. in Math. 4 (1970), 209-223.

, Stabilization of solutions of a caricature of the Fitzhugh-Nagumo equation, Comm. Pure Appl. Math. 36 (1983), 291-324; ibid. 37 (1984), 299-301.

J. Nagumo, S. Arimoto and S. Yoshizawa, An active pulse transmission line simulating nerve axom, Proc. IRE 50 (1964), 2061-2070.

J. Rinzel, Simple equations for active nerve condition and passive neuronal integration, Lectures Math. Life Sci., Vol. 8, Amer. Math. Soc., Providence, R.I., 1976, pp. 125-164. 
D. Terman, Threshold phenomena for a reaction-diffusion system, J. Differential Equations 47 (1983), 406-443.

_,$A$ free-boundary problem arising from a bi-stable reaction-diffusion equation, SIAM J. Math. Anal. 14 (1983), 1107-1129.

DEPARTMENT OF MATHEMATICS, COURANT INSTitute OF MATHEMatical SCIENCES, NEW YORK, NEW YORK 10012

Department of Mathematics, Temple University, Philadelphia, PennSYLVANIA 19122 